\title{
AN INTERPRETATION OF MULTIPLIER IDEALS VIA TIGHT CLOSURE
}

\author{
SHUNSUKE TAKAGI
}

\begin{abstract}
Hara Ha3] and Smith Sm2 independently proved that in a normal $\mathbb{Q}$-Gorenstein ring of characteristic $p \gg 0$, the test ideal coincides with the multiplier ideal associated to the trivial divisor. We extend this result for a pair $(R, \Delta)$ of a normal ring $R$ and an effective $\mathbb{Q}$-Weil divisor $\Delta$ on $\operatorname{Spec} R$. As a corollary, we obtain the equivalence of strongly F-regular pairs and klt pairs.
\end{abstract}

\section{INTRODUCTION}

Recently it turned out that there exists a relation between multiplier ideals and tight closure. Precisely speaking, it was proved that some algebraic statements established by multiplier ideals could also be understood via tight closure, for example, Briançon-Skoda theorem (see [BS], [HH1] and [La]), the problem concerning the growth of symbolic powers of ideals in regular local rings (see [ELS] and [HH3]), etc. The purpose of this paper is to give an interpretation of multiplier ideals via tight closure.

The theory of tight closure was introduced by Hochster and Huneke [HH1, using the Frobenius map in characteristic $p>0$. In this theory, test ideals play a central role. On the other hand, multiplier ideals, for which we have the strong vanishing theorem, are fundamental tools in birational geometry. Hara [Ha3] and Smith [Sm2] independently proved that in a normal $\mathbb{Q}$-Gorenstein ring of characteristic $p \gg 0$, the test ideal coincides with the multiplier ideal associated to the trivial divisor. Since the real worth of multiplier ideals is displayed in considering pairs, we attempt to extend this result for pairs. Here, by a pair, we mean a pair $(R, \Delta)$ of a normal ring $R$ and an effective $\mathbb{Q}$-Weil divisor $\Delta$ on Spec $R$.

Hara generalized the notion of tight closure to that for pairs, which is called $\Delta$ tight closure (cf. [HW, Problem 5.3.2]). Using the $\Delta$-tight closure operation, we introduce the ideal $\tau(R, \Delta)$ associated to a pair $(R, \Delta)$, which is a generalization of the notion of test ideals. We denote by $\mathcal{J}(Y, \Delta)$ the multiplier ideal of $(Y, \Delta)$. Then, our main result is stated as follows:

Theorem 3.2. Let $(R, \mathfrak{m})$ be a normal local ring essentially of finite type over a field of characteristic zero and $\Delta$ an effective $\mathbb{Q}$-Weil divisor on $Y=\operatorname{Spec} R$ such

1991 Mathematics Subject Classification. Primary 13A35, 14B05; Secondary 14B15, 14E15.

Key words and phrases. Multiplier ideal, Kawamata log terminal, tight closure, strongly Fregular, F-singularities of pairs, modulo $p$ reduction. 
that $K_{Y}+\Delta$ is $\mathbb{Q}$-Cartier. Then, in characteristic $p \gg 0$,

$$
\tau(R, \Delta)=\mathcal{J}(Y, \Delta)
$$

When $\Delta=0$, our main theorem coincides with Hara and Smith's result, and they used the strategy which is to reduce to the case where the ring $R$ is quasi-Gorenstein by passing to an index one cover. However we cannot use this strategy, because in general $K_{Y}$ could be a non- $\mathbb{Q}$-Cartier divisor, even if $K_{Y}+\Delta$ is $\mathbb{Q}$-Cartier. So we give the direct proof which does not use an index one cover.

As a corollary of the main theorem, we get the equivalence of "F-singularities of pairs" and "singularities of pairs."

The notions of F-regular and F-pure rings, which are closely related to the theory of tight closure, were defined by Hochster and Huneke HH1 and Hochster and Roberts [HR respectively. Recently it became clear that F-singularities (F-regular and F-pure rings) correspond to singularities arising in birational geometry (Kawamata log terminal and log canonical singularities). See [Ha2], [HW], [MS] and [Sm1]. The notions of Kawamata log terminal (or klt for short) and log canonical (or lc for short) singularities are defined not only for normal rings but also for pairs, and it is these "singularities of pairs" that play a very important role in birational geometry. Therefore Hara and K.-i. Watanabe [HW] generalized the notions of F-singularities to those for pairs, and they conjectured the equivalence of "F-singularities of pairs" and "singularities of pairs." We prove their conjectures.

The ideal $\tau(R, \Delta)$ defines the locus of non-F-regular points of $(R, \Delta)$ in $\operatorname{Spec} R$ under the assumption that $K_{R}+\Delta$ is a $\mathbb{Q}$-Cartier divisor. Likewise, the multiplier ideal $\mathcal{J}(\operatorname{Spec} R, \Delta)$ defines the locus of non-klt points of $(\operatorname{Spec} R, \Delta)$. Hence we obtain the following result as a direct consequence of the main result.

Corollary 3.4 ([ [सW, Conjecture 5.1.1]). Let $(R, \mathfrak{m})$ be a normal local ring essentially of finite type over a field of characteristic zero and $\Delta$ an effective $\mathbb{Q}$-Weil divisor on $Y=\operatorname{Spec} R$ such that $K_{Y}+\Delta$ is $\mathbb{Q}$-Cartier. Then, $(Y, \Delta)$ is klt if and only if $(R, \Delta)$ is of strongly F-regular type.

Acknowledgements. The author wishes to thank Professor Toshiyuki Katsura, his research supervisor, for warm encouragement. He is also grateful to Professor

Kei-ichi Watanabe for various comments and many suggestions, Professor Nobuo Hara for helpful advices and valuable information about the subadditivity theorem for the ideal " $\tau(R, \mathfrak{a})$," and Yasunari Nagai for many discussions about birational geometry.

\section{Preliminaries}

1.1. F-singularities of pairs. First we briefly review definitions and basic properties on "F-singularities of pairs," which were introduced by Hara and K.-i. Watanabe. Refer to [HW] for details. 
Throughout this paper, all rings are commutative Noetherian integral domains with identity. Let $R$ be an integral domain of characteristic $p>0$ and $F: R \rightarrow R$ the Frobenius map which sends $x$ to $x^{p}$. Since $R$ is reduced, we can identify $F: R \rightarrow R$ with the natural inclusion map $R \hookrightarrow R^{1 / p}$. $R$ is called F-finite if $R \hookrightarrow R^{1 / p}$ is a finite map. For example, any algebra essentially of finite type over a perfect field is F-finite. We also remark that if $R$ is F-finite, then $R$ is excellent $\mathrm{Ku}$.

Notation. Let $R$ be a normal domain with quotient field $K$. A $\mathbb{Q}$-Weil divisor $D$ on $Y=\operatorname{Spec} R$ is a linear combination $D=\sum_{i=1}^{r} a_{i} D_{i}$ of irreducible reduced subschemes $D_{i} \subset Y$ of codimension one with rational coefficients $a_{i}$. The round-up and round-down of $D$ is defined by $\lceil D\rceil=\sum_{i=1}^{r}\left\lceil a_{i}\right\rceil D_{i}$ and $\lfloor D\rfloor=\sum_{i=1}^{r}\left\lfloor a_{i}\right\rfloor D_{i}$. We also denote

$$
R(D)=\left\{x \in K \mid \operatorname{div}_{R}(x)+D \geq 0\right\} .
$$

Definition 1.1 ([世W, Definition 2.1]). Let $R$ be an F-finite normal domain of characteristic $p>0$ and $\Delta$ an effective $\mathbb{Q}$-Weil divisor on $\operatorname{Spec} R$.

(1) $(R, \Delta)$ is said to be F-pure if the inclusion map $R \hookrightarrow R((q-1) \Delta)^{1 / q}$ splits as an $R$-module homomorphism for every $q=p^{e}$.

(2) $(R, \Delta)$ is said to be strongly $F$-regular if for every nonzero element $c \in R$, there exists $q=p^{e}$ such that $c^{1 / q} R \hookrightarrow R((q-1) \Delta)^{1 / q}$ splits as an $R$-module homomorphism.

Remark 1.2. (i) $R$ is F-pure (resp. strongly F-regular) if and only if $(R, 0)$ is Fpure (resp. strongly F-regular). Refer to [HH1], [HH2 and [HR for strongly F-regular and F-pure rings. Here we only note that the following implications hold for rings. regular $\Rightarrow$ strongly F-regular $\Rightarrow$ normal and Cohen-Macaulay

$$
\text { F-pure }
$$

(ii) We can replace $R((q-1) \Delta)^{1 / q}$ by $R(\lceil q \Delta\rceil)^{1 / q}$ in the above definition of strong F-regularity.

Basic Properties ([ $[\mathrm{HW}$, Proposition 2.2]). Let $(R, \Delta)$ be as above.

(i) Strong F-regularity implies F-purity.

(ii) $(R, \Delta)$ is strongly F-regular if and only if for every nonzero element $c \in R$, there exists $q^{\prime}$ such that $c^{1 / q} R \hookrightarrow R(q \Delta)^{1 / q}$ splits as an $R$-module homomorphism for all $q=p^{e} \geq q^{\prime}$.

(iii) If $(R, \Delta)$ is strongly F-regular, then $\lfloor\Delta\rfloor=0$.

(iv) If $(R, \Delta)$ is F-pure, then $\lceil\Delta\rceil$ is reduced.

(v) If $(R, \Delta)$ is F-pure (resp. strongly F-regular), so is $\left(R, \Delta^{\prime}\right)$ for every effective $\mathbb{Q}$-Weil divisor $\Delta^{\prime} \leq \Delta$.

Example 1.3 (cf. [Fe, Theorem 2.5] and [HW, Corollary 2.7]). Let $R=$ $k\left[\left[x_{1}, \ldots, x_{n}\right]\right]$ be an $n$-dimensional complete regular local ring over a field $k$ of characteristic $p>0$ and $\Delta=\operatorname{div}_{R}\left(x_{1}^{d_{1}}+\cdots+x_{n}^{d_{n}}\right)$. Assume that $p$ is sufficiently large 
and let $t_{0}=\min \left\{1, \sum_{i=1}^{n} \frac{1}{d_{i}}\right\}$. Then, $(R, t \Delta)$ is strongly F-regular if and only if $t<t_{0}$. If $(R, t \Delta)$ is F-pure, then $t \leq t_{0}$. When $\sum_{i=1}^{n} \frac{1}{d_{i}}>1$, then $\left(R, t_{0} \Delta\right)$ is always F-pure. On the other hand when $\sum_{i=1}^{n} \frac{1}{d_{i}} \leq 1$, then $\left(R, t_{0} \Delta\right)$ is F-pure if $p \equiv 1 \bmod$ $d_{i}$ for every $i=1, \ldots, n$.

The notions of F-regularity and F-purity are also defined for rings of characteristic zero as follows.

Definition 1.4. Let $R$ be a finitely generated normal domain over a field $k$ of characteristic zero and $\Delta$ an effective $\mathbb{Q}$-Weil divisor on Spec $R$. The pair $(R, \Delta)$ is said to be of F-pure type (resp. strongly F-regular type) if there exist a finitely generated $\mathbb{Z}$-subalgebra $A$ of $k$, a finitely generated normal $A$-algebra $R_{A}$ and an effective $\mathbb{Q}$-Weil divisor $\Delta_{A}$ on $\operatorname{Spec} R_{A}$, with a flat structure map $A \rightarrow R_{A}$ such that

(1) $\left(A \rightarrow R_{A}\right) \otimes_{A} k \cong(k \rightarrow R)$ and $\Delta_{A} \otimes_{A} k \cong \Delta$.

(2) $\left(R_{\kappa}, \Delta_{\kappa}\right)$ is F-pure (resp. strongly F-regular) for every closed point $s$ in a dense open subset of Spec $A$, where $\kappa=\kappa(s)$ denotes the residue field of $s \in \operatorname{Spec} A, R_{\kappa}=R_{A} \otimes_{A} \kappa(s)$ and $\Delta_{\kappa}=\Delta_{A} \otimes_{A} \kappa(s)$.

$(R, \Delta)$ is said to be of dense F-pure type if in the above condition (2) "dense open" is replaced by "dense."

Example 1.5. Let $R=k\left[x_{1}, \ldots, x_{n}\right]$ be an $n$-dimensional polynomial ring over a field $k$ of characteristic zero and $\Delta=\operatorname{div}_{R}\left(x_{1}^{d_{1}}+\cdots+x_{n}^{d_{n}}\right)$. Then, $(R, t \Delta)$ is of strongly F-regular type (resp. of dense F-pure type) if and only if $\min \left\{1, \sum_{i=1}^{n} \frac{1}{d_{i}}\right\}>$ $t$ (resp. $\left.\min \left\{1, \sum_{i=1}^{n} \frac{1}{d_{i}}\right\} \geq t\right)$.

1.2. Birational Geometry. We recall definitions and fundamental properties of singularities which appear in the Mori theory, and of multiplier ideal sheaves. Refer to $\mathrm{KM}$ and $\mathrm{La}$ for details.

Let $Y$ be a normal variety over a field of characteristic zero and $\Delta$ a $\mathbb{Q}$-Weil divisor on $Y$ such that $K_{Y}+\Delta$ is $\mathbb{Q}$-Cartier, that is, $r\left(K_{Y}+\Delta\right)$ is a Cartier divisor for some positive integer $r$, where $K_{Y}$ is the canonical divisor of $Y$. Let $f: X \rightarrow Y$ be a resolution of singularities such that $\cup_{i=1}^{s} E_{i}+f_{*}^{-1} \Delta$ has simple normal crossing support, where $\operatorname{Exc}(f)=\cup_{i=1}^{s} E_{i}$ is the exceptional divisor of $f$ and $f_{*}^{-1} \Delta$ is the strict transform of $\Delta$ in $X$. We denote by $K_{X}$ the canonical divisor of $X$. Then, for some integers $b_{1}, \ldots, b_{s}$,

$$
r\left(K_{X}+f_{*}^{-1} \Delta\right) \underset{\text { lin. }}{\sim} f^{*}\left(r\left(K_{Y}+\Delta\right)\right)+\sum_{i=1}^{s} b_{i} E_{i} .
$$

Hence we have

$$
K_{X}+f_{*}^{-1} \Delta \underset{\mathbb{Q} \text {-lin. }}{\sim} f^{*}\left(K_{Y}+\Delta\right)+\sum_{i=1}^{s} a_{i} E_{i},
$$

where $a_{i}=b_{i} / r(i=1, \ldots, s)$. 
Definition 1.6. Under the same notation as above:

(1) We say that the pair $(Y, \Delta)$ is Kawamata log terminal (or klt for short) if $a_{i}>-1$ for every $i=1, \ldots, s$ and $\lfloor\Delta\rfloor \leq 0$.

(2) We say that the pair $(Y, \Delta)$ is $\log$ canonical (or $l c$ for short) if $a_{i} \geq-1$ for every $i=1, \ldots, s$ and the coefficient of $\Delta$ in each irreducible component is less than or equal to one.

(3) The multiplier ideal sheaf $\mathcal{J}(Y, \Delta)$ associated to $\Delta$ is defined to be

$$
\mathcal{J}(Y, \Delta)=f_{*} \mathcal{O}_{X}\left(\left\lceil K_{X}-f^{*}\left(K_{Y}+\Delta\right)\right\rceil\right) .
$$

Remark 1.7. (i) The above definitions do not depend on the choice of a desingularization $f: X \rightarrow Y$.

(ii) When $\Delta$ is effective, $\mathcal{J}(Y, \Delta)$ is indeed an ideal sheaf. However in case $\Delta$ is not effective, it is generally not a submodule of $\mathcal{O}_{Y}$ but a fractional ideal sheaf.

Basic Properties. In the situation of the above definition:

(i) For every $\mathbb{Q}$-Weil divisor $\Delta^{\prime} \leq \Delta$ on $Y, \mathcal{J}\left(Y, \Delta^{\prime}\right) \supseteq \mathcal{J}(Y, \Delta)$.

(ii) For every Cartier divisor $\Delta^{\prime}$ on $Y, \mathcal{J}\left(Y, \Delta+\Delta^{\prime}\right)=\mathcal{J}(Y, \Delta) \otimes_{Y} \mathcal{O}_{Y}\left(-\Delta^{\prime}\right)$.

(iii) The pair $(Y, \Delta)$ is klt if and only if $\mathcal{J}(Y, \Delta) \supseteq \mathcal{O}_{Y}$. In particular when $\Delta$ is effective, $(Y, \Delta)$ is klt if and only if $\mathcal{J}(Y, \Delta)=\mathcal{O}_{Y}$.

Proposition 1.8 (cf. [KM, Proposition 5.20], [La, Example 5.26]). Let $f: X \rightarrow Y$ be a finite covering of normal varieties which is étale in codimension one and $\Delta$ an effective $\mathbb{Q}$-Weil divisor on $Y$. Then

$$
\mathcal{J}(Y, \Delta)=\mathcal{J}\left(X, f^{*} \Delta\right) \cap \mathcal{O}_{Y}
$$

Proposition 1.9 ([DED]). (1) (Restriction Theorem) Let $Y$ be a normal variety, $\Delta$ an effective divisor on $Y$ such that $K_{Y}+\Delta$ is $\mathbb{Q}$-Cartier, and $H$ a reduced Cartier divisor which is not in the support of $\Delta$. Assume that $H$ is a normal variety. Then

$$
\mathcal{J}\left(H,\left.\Delta\right|_{H}\right) \subseteq \mathcal{J}(Y, \Delta) \cdot \mathcal{O}_{H}
$$

(2) (Subadditivity Theorem) Let $Y$ be a non-singular quasi-projective variety, and $\Delta_{1}$ and $\Delta_{2}$ be any two effective $\mathbb{Q}$-divisors on $Y$. Then

$$
\mathcal{J}\left(Y, \Delta_{1}+\Delta_{2}\right) \subseteq \mathcal{J}\left(Y, \Delta_{1}\right) \cdot \mathcal{J}\left(Y, \Delta_{2}\right) .
$$

Example $1.10([\mathrm{La}])$. (1) When $Y$ is a non-singular variety and $\Delta$ is a $\mathbb{Q}$ Weil divisor on $Y$ with simple normal crossing support, then $\mathcal{J}(Y, \Delta)=$ $\mathcal{O}_{Y}(-\lfloor\Delta\rfloor)$.

(2) Let $Y=\mathbb{C}^{n}$ with coordinates $x_{1}, \ldots, x_{n}$ and $\Delta=\operatorname{div}_{Y}\left(x_{1}^{d_{1}}+\cdots+x_{n}^{d_{n}}\right)$. Then, $(Y, t \Delta)$ is klt (resp. lc) if and only if $\min \left\{1, \sum_{i=1}^{n} \frac{1}{d_{i}}\right\}>t$ (resp. $\left.\min \left\{1, \sum_{i=1}^{n} \frac{1}{d_{i}}\right\} \geq t\right)$.

(3) Let $Y=\mathbb{C}^{d}$ with coordinates $x_{1}, \ldots, x_{d}$ and $\Delta=\operatorname{div}_{Y}\left(x_{1}^{d+1}+\cdots+x_{d}^{d+1}\right)$. Then, $\mathcal{J}\left(Y, \frac{d}{d+1} \Delta\right)=\left(x_{1}, \ldots, x_{d}\right)$. 


\section{2. $\Delta$-TIGHT CLOSURE}

In this section, we introduce the notion of $\Delta$-tight closure which is suggested in [HW, Problem 5.3.2] and see that the $\Delta$-tight closure operation satisfies properties similar to those of the "usual" tight closure operation (see [Hu] for the theory of "usual" tight closure). Moreover, using the $\Delta$-tight closure operation, we define the ideal $\tau(R, \Delta)$, which is a generalization of the "usual" test ideal.

Notation. Let $R$ be a normal domain of characteristic $p>0$ and $\Delta$ an effective $\mathbb{Q}$-Weil divisor on $\operatorname{Spec} R$.

- We always use the letter $q$ (resp. $\left.q^{\prime}, q^{\prime \prime}\right)$ for a power $p^{e}$ (resp. $p^{e^{\prime}}, p^{e^{\prime \prime}}$ ) of $p$.

- For any ideal $I$ in $R$, we denote by $I^{[q]}$ the ideal of $R$ generated by the $q$ th powers of elements of $I$.

- For any divisorial ideal $J$ of $\mathrm{R}$ (i.e., $J=R(D)$ for some unique integral Weil divisor $D$ ), we denote by $J^{(m)}$ the reflexive hull of $J^{m}$. If $J=R(D)=$ $R(\lfloor D\rfloor)$, then $J^{(m)}=R(m\lfloor D\rfloor)$.

- The notation ${ }^{e} R((q-1) \Delta)$ denotes $R((q-1) \Delta)$ itself, but viewed as an $R$-module via the $e$-times Frobenius map $F^{e}: R \rightarrow R((q-1) \Delta)$.

- When $(R, \mathfrak{m})$ is local, we denote by $E_{R}$ the injective hull of the residue field $R / \mathfrak{m}$.

Definition 2.1 (cf. [HW, Problem 5.3.2]). Let $N \subseteq M$ be modules over an F-finite normal domain $R$ of characteristic $p>0$ and $\Delta$ an effective $\mathbb{Q}$-Weil divisor on Spec $R$. We denote by $F^{e}: M=M \otimes_{R} R \rightarrow M \otimes_{R}{ }^{e} R((q-1) \Delta)$ the $e$-times Frobenius map induced on $M$ which sends $z \in M$ to $z^{q}:=F^{e}(z)=z \otimes 1 \in M \otimes_{R}{ }^{e} R((q-1) \Delta)$. Set $N_{M}^{[q] \Delta}:=\operatorname{Im}\left(F^{e}(N) \rightarrow F^{e}(M)\right)$. Then the $\Delta$-tight closure $N_{M}^{* \Delta} \subseteq M$ of $N$ in $M$ is defined as follows: $z \in N_{M}^{* \Delta}$ if and only if there exists a nonzero element $c \in R$ such that $c z^{q}:=z \otimes c \in N_{M}^{[q] \Delta}$ for all $q=p^{e} \gg 0$.

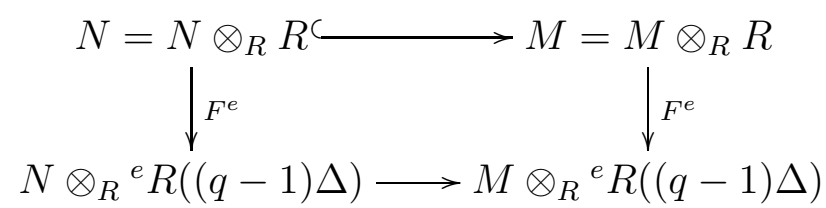

Moreover, the finitistic $\Delta$-tight closure $N_{M}^{* \Delta f g} \subseteq M$ of $N$ in $M$ is defined to be $N_{M}^{* \Delta f g}:=\cup_{M^{\prime}} N_{M^{\prime}}^{* \Delta}$, where $M^{\prime}$ runs through all finitely generated $R$-submodules of $M$ which contain $N$.

Remark 2.2. (i) When $\Delta=0, \Delta$-tight closure coincides with "usual" tight closure.

(ii) In general, $N_{M}^{* \Delta} \subsetneq\left(N_{M}^{* \Delta}\right)_{M}^{* \Delta}$. In this sense, the $\Delta$-tight closure operation is not a "closure operation."

(iii) Let $I$ be an ideal in $R$. Then, $I_{R}^{[q] \Delta}=I^{[q]} R((q-1) \Delta)$.

(iv) We can replace ${ }^{e} R((q-1) \Delta)$ by ${ }^{e} R(\lceil q \Delta\rceil)$ in the above definition. 
(v) In general, $N_{M}^{* \Delta f g} \subseteq N_{M}^{* \Delta}$. If $M$ itself is finitely generated, then $N_{M}^{* \Delta f g}=$ $N_{M}^{* \Delta}$.

Basic Properties. In the situation of the above definition,

(i) $N \subseteq N_{M}^{* \Delta}$.

(ii) $N_{M}^{* \Delta} / N=0_{M / N}^{* \Delta}$.

(iii) For any effective $\mathbb{Q}$-Weil divisor $\Delta^{\prime} \leq \Delta$ on $\operatorname{Spec} R, N_{M}^{* \Delta^{\prime}} \subseteq N_{M}^{* \Delta}$.

(iv) For any effective Cartier divisor $\Delta^{\prime}$ on Spec $R, N_{M}^{*\left(\Delta+\Delta^{\prime}\right)}=N_{M}^{* \Delta}: R\left(-\Delta^{\prime}\right)$.

(v) If $(R, \Delta)$ is strongly F-regular, then $I^{* \Delta}=I$ for every ideal $I$ in $R$.

Strong F-regularity can be characterized via $\Delta$-tight closure.

Lemma 2.3. Let $(R, \mathfrak{m})$ be an F-finite normal local ring of characteristic $p>0$ and $\Delta$ an effective $\mathbb{Q}$-Weil divisor on $\operatorname{Spec} R$. Then $(R, \Delta)$ is strongly F-regular if and only if $0_{E}^{* \Delta}=0$, where $E=E(R / \mathfrak{m})$ is the injective hull of the residue field $R / \mathfrak{m}$.

Proof. The proof is essentially the same as that for the no boundary case Ha1, Proposition 2.1].

Assume that $(R, \Delta)$ is strongly F-regular and fix any element $z \in 0_{E}^{* \Delta}$. Then there exists a nonzero element $c \in R$ such that $c z^{q}:=c F^{e}(z)=0$ for all $q=p^{e} \gg 0$. Let

$$
\phi_{c}^{(e)}: \operatorname{Hom}_{R}\left(R((q-1) \Delta)^{1 / q}, R\right) \rightarrow \operatorname{Hom}_{R}(R, R)=R
$$

be an $R$-module homomorphism induced by the $R$-linear map $R \stackrel{c^{1 / q}}{\longrightarrow} R((q-1) \Delta)^{1 / q}$ for each $q=p^{e}$. Since $(R, \Delta)$ is strongly F-regular, $\phi_{c}^{(e)}$ is surjective for all $q=p^{e} \gg$ 0 . Since the $R$-module homomorphism $c F^{e}: E \rightarrow E \otimes_{R}{ }^{e} R((q-1) \Delta)$ which sends $z$ to $c z^{q}$ is the Matlis dual of $\phi_{c}^{(e)}, c F^{e}$ is injective for every $q=p^{e} \gg 0$. Hence we have $z=0$.

Conversely, suppose that $0_{E}^{* \Delta}=0$, and fix any nonzero element $c \in R$. If $z$ is a nonzero element of the socle $(0: \mathfrak{m})_{E}$ of $E$, then there exists $q=p^{e}$ such that $c F^{e}(z) \neq 0$. Since $(0: \mathfrak{m})_{E}$ is an one-dimensional $R / \mathfrak{m}$-vector space, we can take $q$ which works for every $z \in(0: \mathfrak{m})_{E}$. Then $c F^{e}$ is injective on $(0: \mathfrak{m})_{E}$. Since $E$ is an essential extension of $(0: \mathfrak{m})_{E}, c F^{e}$ itself is injective. Taking the Matlis dual of $c F^{e}$, we know that $\phi_{c}^{(e)}$ is surjective, namely $(R, \Delta)$ is strongly F-regular.

We introduce $\Delta$-test elements which are very useful to show the propositions about $\Delta$-tight closure. When $\Delta$ is zero, a $\Delta$-test element is nothing but a test element in the theory of "usual" tight closure.

Definition 2.4. Let $R$ be an F-finite normal domain of characteristic $p>0$ and $\Delta$ an effective $\mathbb{Q}$-Weil divisor on $\operatorname{Spec} R$. A nonzero element $c \in R$ is called a $\Delta$-test element if for each ideal $I$ in $R, x \in I^{* \Delta}$ if and only if $c x^{q} \in I^{[q]} R(\lceil q \Delta\rceil)$ for all $q=p^{e}$.

By the following lemma, which is a generalization of [HH2, Theorem 3.3], $\Delta$-test elements always exist. 
Lemma 2.5. Let $R$ be an F-finite normal domain of characteristic $p>0$. Let $c \in R$ be any nonzero element such that the localization $R_{c}$ with respect to $c$ is strongly F-regular.

(1) For every effective divisor $\Delta$ on $R,(R, \Delta)$ is strongly F-regular if and only if there exists $q=p^{e}$ such that $c^{1 / q} R \hookrightarrow R(\lceil q \Delta\rceil)^{1 / q}$ splits as an $R$-module homomorphism.

(2) For every effective divisor $\Delta$ on $R, c^{n}$ is a $\Delta$-test element for some positive integer $n$.

Proof. Let $d \in R$ be any nonzero element.

Claim 1. For some $q^{\prime}$ and sufficiently large $q^{\prime \prime}$, there exists an $R^{1 / q q^{\prime \prime}}$-module homomorphism

$$
R\left(\left\lceil q q^{\prime} q^{\prime \prime} \Delta\right\rceil\right)^{1 / q q^{\prime} q^{\prime \prime}} \rightarrow R\left(\left\lceil q q^{\prime \prime} \Delta\right\rceil\right)^{1 / q q^{\prime \prime}}, \quad d^{1 / q q^{\prime} q^{\prime \prime}} \mapsto c^{1 / q} .
$$

Proof of Claim 1. Since $R_{c}$ is strongly F-regular, by the proof of [HH2, Theorem 3.3], for some $q^{\prime}$ and sufficiently large $q^{\prime \prime}$, there exists an $R$-module homomorphism

$$
R^{1 / q^{\prime}} \rightarrow R, \quad d^{1 / q^{\prime}} \mapsto c^{q^{\prime \prime}}
$$

Tensoring with $R\left(\left\lceil q q^{\prime \prime} \Delta\right\rceil\right)$ and taking $q q^{\prime \prime}$-th roots, for each $q=p^{e}$, we get an $R^{1 / q q^{\prime \prime}}$-linear map

$$
R\left(\left\lceil q q^{\prime} q^{\prime \prime} \Delta\right\rceil\right)^{1 / q q^{\prime} q^{\prime \prime}} \rightarrow R\left(\left\lceil q q^{\prime \prime} \Delta\right\rceil\right)^{1 / q q^{\prime \prime}}, \quad d^{1 / q q^{\prime} q^{\prime \prime}} \mapsto c^{q^{\prime \prime} / q q^{\prime \prime}}=c^{1 / q} .
$$

First we will show (1). Suppose that there exists $q=p^{e}$ such that $c^{1 / q} R \hookrightarrow$ $R(\lceil q \Delta\rceil)^{1 / q}$ splits as an $R$-module homomorphism, that is, there exists an $R$-linear map $R(\lceil q \Delta\rceil)^{1 / q} \rightarrow R$ sending $c^{1 / q}$ to 1 . We may replace $q^{\prime \prime}$ in Claim 1 suitably (for example, $q^{\prime \prime}=q^{n}$ for some positive integer $n$ ), and then there exists an $R$-module homomorphism $R\left(\left\lceil q^{\prime \prime} \Delta\right\rceil\right)^{1 / q^{\prime \prime}} \rightarrow R$ which sends 1 to 1 . Tensoring this with $R(\lceil q \Delta\rceil)$ and taking $q$-th roots, we obtain an $R^{1 / q}$-linear map $R\left(\left\lceil q q^{\prime \prime} \Delta\right\rceil\right)^{1 / q q^{\prime \prime}} \rightarrow R(\lceil q \Delta\rceil)^{1 / q}$ which sends 1 to 1 . By composing these maps with the $R^{1 / q q^{\prime \prime}}$-linear map in Claim 1 , we get the following $R$-module homomorphism

$$
\begin{gathered}
R\left(\left\lceil q q^{\prime} q^{\prime \prime} \Delta\right\rceil\right)^{1 / q q^{\prime} q^{\prime \prime}} \rightarrow R\left(\left\lceil q q^{\prime \prime} \Delta\right\rceil\right)^{1 / q q^{\prime \prime}} \rightarrow R(\lceil q \Delta\rceil)^{1 / q} \rightarrow R, \\
d^{1 / q q^{\prime} q^{\prime \prime}} \longmapsto c^{1 / q} \longmapsto c^{1 / q} \longmapsto 1 .
\end{gathered}
$$

This establishes (1).

Next we will prove (2). When $d=1$, then we can take $p$ as $q^{\prime}$ in the proof of Claim 1, namely, there exists an $R$-module homomorphism

$$
h: R^{1 / p} \rightarrow R, \quad 1 \mapsto c^{q^{\prime \prime}} .
$$

We replace $c^{q^{\prime \prime}}$ by $c$, and then it is enough to show that $c^{3}$ is a $\Delta$-test element.

Claim 2. For every $q=p^{e}$, there exists an $R$-module homomorphism

$$
g_{e}: R(q\lceil\Delta\rceil)^{1 / q} \rightarrow R(\lceil\Delta\rceil), \quad 1 \mapsto c^{2} .
$$


Proof of Claim 2. When $q=p$, then tensoring $h$ with $R(\lceil\Delta\rceil)$, we obtain an $R$-linear map

$$
h_{1}: R(p\lceil\Delta\rceil)^{1 / p} \rightarrow R(\lceil\Delta\rceil) \quad 1 \mapsto c .
$$

Hence we may set $g_{1}=c \cdot h_{1}$. Suppose that the assertion holds for $q=p^{e}$. Then, by tensoring with $R((p-1)\lceil\Delta\rceil)$ and taking $p$-th roots, we obtain an $R^{1 / p}$-linear map $R(p q\lceil\Delta\rceil)^{1 / p q} \rightarrow R(p\lceil\Delta\rceil)^{1 / p}$ which sends 1 to $c^{2 / p}$. We may compose this with an $R^{1 / p}$-module homomorphism $R(p\lceil\Delta\rceil)^{1 / p} \rightarrow R(p\lceil\Delta\rceil)^{1 / p}$ which sends 1 to $c^{(p-2) / p}$, and then with $h_{1}$.

$$
\begin{gathered}
R(p q\lceil\Delta\rceil)^{1 / p q} \rightarrow R(p\lceil\Delta\rceil)^{1 / p} \rightarrow R(p\lceil\Delta\rceil)^{1 / p} \rightarrow R, \\
1 \longmapsto c^{2 / p} \longmapsto c \longmapsto c^{2} .
\end{gathered}
$$

This is a required homomorphism for $p q=p^{e+1}$.

Thanks to Claim 2, tensoring $g_{e^{\prime \prime}}$ with $R(\lceil q \Delta\rceil-\lceil\Delta\rceil)$ and taking $q$-th roots, we obtain an $R^{1 / q}$-module homomorphism

$$
R\left(\left\lceil q q^{\prime \prime} \Delta\right\rceil\right)^{1 / q q^{\prime \prime}} \rightarrow R(\lceil q \Delta\rceil)^{1 / q}, \quad 1 \mapsto c^{2 / q} .
$$

Therefore, by Claim 1, there exists an $R^{1 / q_{-}}$linear map

$$
\begin{gathered}
R\left(\left\lceil q q^{\prime} q^{\prime \prime} \Delta\right\rceil\right)^{1 / q q^{\prime} q^{\prime \prime}} \rightarrow R\left(\left\lceil q q^{\prime \prime} \Delta\right\rceil\right)^{1 / q q^{\prime \prime}} \rightarrow R(\lceil q \Delta\rceil)^{1 / q}, \\
d^{1 / q q^{\prime} q^{\prime \prime}} \longmapsto c^{1 / q} \longmapsto c^{3 / q} .
\end{gathered}
$$

Thus, $d x^{q q^{\prime} q^{\prime \prime}} \in I^{\left[q q^{\prime} q^{\prime \prime}\right]} R\left(\left\lceil q q^{\prime} q^{\prime \prime} \Delta\right\rceil\right)$ implies $c^{3} x^{q} \in I^{[q]} R(\lceil q \Delta\rceil)$. It follows that $c^{3}$ is a $\Delta$-test element.

Now we define the ideal $\tau(R, \Delta)$.

Definition 2.6. Let $R$ be an F-finite normal domain of characteristic $p>0$ and $\Delta$ an effective $\mathbb{Q}$-Weil divisor on $Y=\operatorname{Spec} R$. Then the ideal $\tau(R, \Delta)$ is defined to be $\tau(R, \Delta):=\bigcap_{M} \operatorname{Ann}_{R}\left(0_{M}^{* \Delta}\right) \subseteq R$, where $M$ runs through all finitely generated $R$-modules.

Remark 2.7. When $\Delta=0, \tau(R, \Delta)$ coincides with the "usual" test ideal which is generated by $\left(\Delta\right.$-)test elements. However even if $K_{Y}+\Delta$ is $\mathbb{Q}$-Cartier, $\tau(R, \Delta)$ may not be generated by $\Delta$-test elements. Therefore We do not call $\tau(R, \Delta)$ the $\Delta$-test ideal.

Basic Properties. In the situation of the above definition:

(i) For any effective $\mathbb{Q}$-Weil divisor $\Delta^{\prime} \leq \Delta$ on Spec $R$, we have $\tau\left(R, \Delta^{\prime}\right) \supseteq$ $\tau(R, \Delta)$.

(ii) For any effective Cartier divisor $\Delta^{\prime}$ on $\operatorname{Spec} R$, we have $\tau\left(R, \Delta+\Delta^{\prime}\right)=$ $\tau(R, \Delta) \otimes_{R} R\left(-\Delta^{\prime}\right)$.

(iii) If $(R, \Delta)$ is strongly F-regular, then $\tau(R, \Delta)=R$.

Theorem 2.8. Let $R$ be an F-finite normal domain of characteristic $p>0$ and $\Delta$ an effective $\mathbb{Q}$-Weil divisor on $Y=\operatorname{Spec} R$. Let $E=\bigoplus_{\mathfrak{m}} E(R / \mathfrak{m})$, where $\mathfrak{m}$ runs through all maximal ideals of $R$. 
(1)

$$
\tau(R, \Delta)=\bigcap_{I}\left(I: I^{* \Delta}\right)=\operatorname{Ann}_{R}\left(0_{E}^{* \Delta f g}\right),
$$

where the intersection in the middle term is taken over all ideals $I$ in $R$.

(2) If $K_{Y}+\Delta$ is a $\mathbb{Q}$-Cartier divisor, then

$$
\tau(R, \Delta)=\operatorname{Ann}_{R}\left(0_{E}^{* \Delta}\right)
$$

Proof. By the same argument as in the proof of [HH1, Proposition 8.23], we see that $\bigcap_{M} \operatorname{Ann}_{R}\left(0_{M}^{* \Delta}\right)=\bigcap_{I}\left(I: I^{* \Delta}\right)=\operatorname{Ann}_{R}\left(0_{E}^{* \Delta f g}\right)$. So we will prove (2). We may assume that $(R, \mathfrak{m})$ is local. Since $0_{E}^{* \Delta} \supseteq 0_{E}^{* \Delta f g}$, it is enough to show that $\operatorname{Ann}_{R}\left(0_{E}^{* \Delta}\right) \supseteq \bigcap_{I}\left(I: I^{* \Delta}\right)$ under the assumption that $K_{Y}+\Delta$ is $\mathbb{Q}$-Cartier.

We use the same strategy as that of [Ha3]. For a sequence of elements $\mathbf{x}=$ $x_{1}, \ldots, x_{d}$ of $R$ and a positive integer $t$, we write $\mathbf{x}^{t}=x_{1}^{t}, \ldots, x_{d}^{t}$. For an $R$-module $M$, we denote

$$
\mathcal{K}(\mathbf{x}, t, M):=\operatorname{Ker}\left(\frac{M}{(\mathbf{x}) M} \stackrel{\left(x_{1} \cdots x_{d}\right)^{t-1}}{\longrightarrow} \frac{M}{\left(\mathbf{x}^{t}\right) M}\right) .
$$

We also denote

$$
\mathcal{K}(\mathbf{x}, \infty, M):=\bigcup_{t \in \mathbb{N}} \mathcal{K}(\mathbf{x}, t, M) .
$$

Claim. Let $(R, \mathfrak{m})$ be a $d$-dimensional normal local ring of characteristic $p>0$ and $J \subseteq R$ a divisorial ideal. Let $\mathbf{x}=x_{1}, \ldots, x_{d}$ be a system of parameters of $R$. Suppose that there exist a nonzero element $c \in R$ and integer $t_{0} \geq 2$ such that

$$
c \mathcal{K}\left(\mathbf{x}^{q s}, \infty, J^{[q]} R((q-1) \Delta)\right) \subseteq \mathcal{K}\left(\mathbf{x}^{q s}, t_{0}, J^{[q]} R((q-1) \Delta)\right)
$$

for all $s \geq 1$ and $q=p^{e} \gg 0$. Then $\operatorname{Ann}_{R}\left(0_{H_{\mathfrak{m}}^{d}(J)}^{* \Delta}\right) \supseteq \bigcap_{I}\left(I: I^{* \Delta}\right)$.

Proof of Claim. Let $\xi \in 0_{H_{\mathfrak{m}}^{d}(J)}^{* \Delta}$, i.e., there exists a nonzero element $d \in R$ such that $d \xi^{q}=0 \in H_{\mathfrak{m}}^{d}(J) \otimes_{R}{ }^{e} R((q-1) \Delta)$ for every $q=p^{e} \gg 0$. Note that $H_{\mathfrak{m}}^{d}(J) \cong$ $\underset{\lim }{\longrightarrow} J /\left(\mathbf{x}^{t}\right) J$, where the direct limit map $J /\left(\mathbf{x}^{t}\right) J \rightarrow J /\left(\mathbf{x}^{t+1}\right) J$ is the multiplication $\overrightarrow{\text { by }} x_{1} x_{2} \cdots x_{d}$. Therefore $\xi$ is represented by $z \bmod \left(\mathbf{x}^{s}\right) J \in J /\left(\mathbf{x}^{s}\right) J$ for some $z \in J$ and $s \geq 1$. We may assume that $s=1$.

Now the natural map

$$
\frac{J}{\left(\mathbf{x}^{t}\right) J} \otimes_{R}{ }^{e} R((q-1) \Delta) \rightarrow \frac{J^{[q]} R((q-1) \Delta)}{\left(\mathbf{x}^{q t}\right) J^{[q]} R((q-1) \Delta)}
$$

induces a map

$$
H_{\mathfrak{m}}^{d}(J) \otimes_{R}{ }^{e} R((q-1) \Delta) \rightarrow \lim _{\longrightarrow} \frac{J^{[q]} R((q-1) \Delta)}{\left(\mathbf{x}^{q t}\right) J[q] R((q-1) \Delta)}
$$

which sends $\xi^{q}$ to the class of $z^{q} \bmod \left(\mathbf{x}^{q}\right) J^{[q]} R((q-1) \Delta)$. Thus, for every $q=p^{e} \gg 0$, class of $d z^{q} \bmod \left(\mathbf{x}^{q}\right) J^{[q]} R((q-1) \Delta)=0 \in \underset{\lim }{\longrightarrow} \frac{J^{[q]} R((q-1) \Delta)}{\left(\mathbf{x}^{q t}\right) J^{[q]} R((q-1) \Delta)}$, 
whence $d z^{q} \in \mathcal{K}\left(\mathbf{x}^{q}, \infty, J^{[q]} R((q-1) \Delta)\right)$. By our assumption, we have $c d z^{q} \in$ $\mathcal{K}\left(\mathbf{x}^{q}, t_{0}, J^{[q]} R((q-1) \Delta)\right)$, namely,

$$
c d z^{q}\left(x_{1} \cdots x_{d}\right)^{q\left(t_{0}-1\right)} \in\left(\mathbf{x}^{q t_{0}}\right) J^{[q]} R((q-1) \Delta)
$$

for every $q=p^{e} \gg 0$. It implies that $z\left(x_{1} \cdots x_{d}\right)^{\left(t_{0}-1\right)} \in\left(\left(\mathbf{x}^{t_{0}}\right) J\right)^{* \Delta}$. Fix any element $a \in \bigcap_{I}\left(I: I^{* \Delta}\right)$. Then $a z\left(x_{1} \cdots x_{d}\right)^{\left(t_{0}-1\right)} \in\left(\mathbf{x}^{t_{0}}\right) J$. Thus it follows that

$$
a \xi=\text { class of } a z \bmod (\mathbf{x}) J=0 \in \underset{\lim }{\longrightarrow} J /\left(\mathbf{x}^{t}\right) J .
$$

Hence the element $a$ is contained in $\operatorname{Ann}_{R}\left(\left(0_{H_{\mathfrak{m}}^{d}(J)}^{* \Delta}\right)\right.$.

Let $J=R(D) \subset R$ be a divisorial ideal which is isomorphic to the canonical module $\omega_{R}$ as an $R$-module. Now we will prove that there exist a nonzero element $d \in R$ and a system of parameters $\mathbf{x}=x_{1}, \ldots, x_{d}$ of $R$ such that

$$
d \mathcal{K}\left(\mathbf{x}^{q s}, \infty, J^{[q]} R((q-1) \Delta)\right) \subseteq \mathcal{K}\left(\mathbf{x}^{q s}, 2, J^{[q]} R((q-1) \Delta)\right)
$$

for all $s \geq 1$ and $q=p^{e} \gg 0$. Since $r(D+\Delta)$ is a Cartier divisor for some positive integer $r$, let $R(r(D+\Delta))=y R$. Fix any $a \in R(-r \Delta)$, and let $x_{1}:=a y \in J$. Then, by Wi, Lemma 4.3], there exist an element $x_{2} \in R$ which is not in any minimal prime divisor of $x_{1}$ and $c \in J$ such that $x_{2}^{n} J^{(n)} \subseteq c^{n} R$ for all $n>0$. The sequence $x_{1}, x_{2}$ can be extended to a system of parameters $\mathbf{x}=x_{1}, \ldots, x_{d}$ for $R$. Now given any power $q=p^{e}$, write $q-1=k r+i$ for integers $k$ and $i$ with $0 \leq i \leq r-1$. Then we have

$$
\begin{aligned}
c^{r} x_{2}^{q} R(k r D+(q-1) \Delta) & \subseteq c^{r} x_{2}^{k r}\left(J^{(k r)} \otimes_{R} R((q-1) \Delta)\right)^{* *} \\
& \subseteq c^{k r+r} R((q-1) \Delta) \\
& \subseteq J^{[q]} R((q-1) \Delta),
\end{aligned}
$$

where ()$^{* *}$ denotes the reflexive hull. Since $x_{1}^{q} \in J^{[q]}$, this implies that

$$
c^{r}\left(x_{1} \cdots x_{i-1} x_{i+1} \cdots x_{d}\right)^{q s} R(k r D+(q-1) \Delta) \subseteq J^{[q]} R((q-1) \Delta)
$$

for every $s \geq 1$ and $i=1, \ldots, n$. Therefore, letting $c_{1}=c^{r}$, we have

$$
\begin{aligned}
& c_{1} \cdot \operatorname{Im}\left(H^{d-1}\left(\mathrm{x}^{q s t} ; \frac{R(k r D+(q-1) \Delta)}{J^{[q]} R((q-1) \Delta)}\right)\right. \\
&\left.\rightarrow H^{d-1}\left(\mathbf{x}^{q s t+q s} ; \frac{R(k r D+(q-1) \Delta)}{J^{[q]} R((q-1) \Delta)}\right)\right)=0
\end{aligned}
$$

for all $s, t \geq 1$. On the other hand, let $c^{\prime} \in R$ be a test element (By Lemma 2.5, such $c^{\prime}$ always exists). If $z \in R(i \Delta)$ is an element such that $z \bmod \left(\mathbf{x}^{q s}\right) R(i \Delta) \in$ $\mathcal{K}\left(\mathbf{x}^{q s}, \infty, R(i \Delta)\right)$, then

$$
a z \in\left(x_{1}^{q s t}, \ldots, x_{n}^{q s t}\right):\left(x_{1} \cdots x_{n}\right)^{q s(t-1)}
$$

for some $t \geq 1$, so $a z \in\left(x_{1}^{q s}, \ldots, x_{n}^{q s}\right)^{*}$ by colon-capturing HH1. Hence, letting $c_{2}=a \cdot c^{\prime}$, we have

$$
c_{2} \mathcal{K}\left(\mathbf{x}^{q s}, \infty, R(k r D+(q-1) \Delta)\right) \cong c_{2} \mathcal{K}\left(\mathbf{x}^{q s}, \infty, R(i \Delta)\right)=0 .
$$


Thus, applying [Ha3, Lemma A.3] to the exact sequence

$$
0 \rightarrow J^{[q]} R((q-1) \Delta) \rightarrow R(k r D+(q-1) \Delta) \rightarrow \frac{R(k r D+(q-1) \Delta)}{J^{[q]} R((q-1) \Delta)} \rightarrow 0,
$$

we see that

$$
c_{1} c_{2} \mathcal{K}\left(\mathbf{x}^{q s}, \infty, J^{[q]} R((q-1) \Delta)\right) \subseteq \mathcal{K}\left(\mathbf{x}^{q s}, 2, J^{[q]} R((q-1) \Delta)\right)
$$

for all $s, t \geq 1$. Thanks to the claim, we obtain the assertion.

Remark 2.9. Theorem 2.8 implies that under the condition that $R$ is complete and $K_{Y}+\Delta$ is $\mathbb{Q}$-Cartier, the finitistic $\Delta$-tight closure of zero coincides with the $\Delta$-tight closure of zero in $E$. In case $\Delta=0$, this holds without the assumption that $R$ is complete (See $[\mathrm{AM}],[\mathrm{Mc}$ and $[\mathrm{Sm} 2]$ ). Hence we believe that this coincidence of the finitistic $\Delta$-tight closure and the $\Delta$-tight closure is true, even if $R$ is not necessarily complete.

By Lemma 2.3, if $K_{R}+\Delta$ is $\mathbb{Q}$-Cartier, then $\tau(R, \Delta)$ defines the locus of non-Fregular points of $(R, \Delta)$ in $\operatorname{Spec} R$.

Corollary 2.10. Let $(R, \mathfrak{m})$ be an F-finite normal local ring of characteristic $p>0$ and $\Delta$ an effective $\mathbb{Q}$-Weil divisor on $\operatorname{Spec} R$ such that $K_{Y}+\Delta$ is $\mathbb{Q}$-Cartier. Then $(R, \Delta)$ is strongly F-regular if and only if $\tau(R, \Delta)=R$, which is equivalent to the condition that $I^{* \Delta}=I$ for every ideal $I$ in $R$.

From now on, we treat only the case where $K_{R}+\Delta$ is $\mathbb{Q}$-Cartier, whence $\tau(R, \Delta)=$ $\operatorname{Ann}_{R}\left(0_{E}^{* \Delta}\right)$. The following proposition corresponds to Proposition 1.8 .

Proposition 2.11. Let $(R, \mathfrak{m}) \hookrightarrow(S, \mathfrak{n})$ be a finite local homomorphism of F-finite normal local rings of characteristic $p>0$ which is étale in codimension one. Let $\Delta_{R}$ be an effective $\mathbb{Q}$-Weil divisor on Spec $R$ such that $K_{R}+\Delta_{R}$ is $\mathbb{Q}$-Cartier, and $\Delta_{S}$ be the pullback of $\Delta_{R}$ by the induced morphism $\pi: \operatorname{Spec} S \rightarrow \operatorname{Spec} R$. Assume that $\operatorname{deg} \pi$ is not divisible by $p$. Then

$$
\tau\left(R, \Delta_{R}\right)=\tau\left(S, \Delta_{S}\right) \cap R
$$

Proof. Note that, by [KM, Proposition 5.7], $R$ is a direct summand of $S$ as an $R$-module. Therefore we consider $E_{R}$ as a direct summand of $E_{S}=S \otimes_{R} E_{R}$. If $\xi \in 0_{E_{R}}^{* \Delta_{R}}$, then there exists a nonzero element $c \in R$ such that $c \xi^{q}=0$ in $E_{R} \otimes_{R}{ }^{e} R\left((q-1) \Delta_{R}\right)$ for all $q=p^{e} \gg 0$, so $c \xi^{q}=0$ in $E_{S} \otimes_{R}{ }^{e} R\left((q-1) \Delta_{R}\right)=$ $E_{S} \otimes_{S} e S\left((q-1) \Delta_{S}\right)$ (this equality follows from the assumption that $R \hookrightarrow S$ is étale in codimension one). This implies that $\tau\left(S, \Delta_{S}\right) \cap R \subseteq \tau\left(R, \Delta_{R}\right)$.

Conversely, let $c$ be a nonzero element of $\tau\left(R, \Delta_{R}\right)$, and fix any nonzero element $d \in R$. Let $F_{R}^{e}: E_{R} \rightarrow E_{R} \otimes_{R}{ }^{e} R\left((q-1) \Delta_{R}\right)\left(\operatorname{resp} . F_{S}^{e}: E_{S} \rightarrow E_{S} \otimes_{S}{ }^{e} S\left((q-1) \Delta_{S}\right)\right)$ be the $e$-times Frobenius map induced on $E_{R}\left(\right.$ resp. $\left.E_{S}\right)$. Then $c \cdot \cap_{e>e^{\prime}} \operatorname{Ker} d F_{R}^{e}=0$ for every $q^{\prime}=p^{e^{\prime}}$. Since $E_{R}$ is Artinian, there exists $q^{\prime \prime}=p^{e^{\prime \prime}}$ such that $\underset{e \geq e^{\prime}}{\cap} \operatorname{Ker} d F_{R}^{e}=$ $\bigcap_{e^{\prime \prime} \geq e \geq e^{\prime}} \operatorname{Ker} d F_{R}^{e}$. As in (Step 1) of the proof of Theorem 2.13, for every $e^{\prime \prime} \geq e \geq e^{\prime}$, 
there exist $c_{e} \in R$ and an $R$-module homomorphism $\phi_{e}{ }^{\prime}: R((q-1) \Delta)^{1 / q} \rightarrow R$ sending $d^{1 / q}$ to $c_{e}$ such that $\sum_{e^{\prime \prime} \geq e \geq e^{\prime}} c_{e}=c$. Since $R \hookrightarrow S$ is étale in codimension one, by tensoring this homomorphism with $E_{S}$ over $R$, we get the following commutative diagram (cf. [HW, Theorem 4.8])

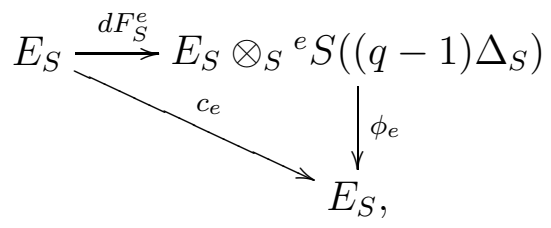

where the $S$-linear map $\phi_{e}: E_{S} \otimes_{S}{ }^{e} S\left((q-1) \Delta_{S}\right) \rightarrow E_{S}$ sends $z \otimes d$ to $c_{e} z$. Therefore $\operatorname{Ker} d F_{S}^{e} \subseteq\left(0: c_{e}\right)_{E_{S}}$ for every $e^{\prime \prime} \geq e \geq e^{\prime}$. Since $\sum_{e^{\prime \prime} \geq e \geq e^{\prime}} c_{e}=c$, we have $\bigcap_{e^{\prime \prime} \geq e \geq e^{\prime}} \operatorname{Ker} d F_{S}^{e} \subseteq(0: c)_{E_{S}}$, and hence $c \cdot \bigcap_{e \geq e^{\prime}} \operatorname{Ker} d F_{S}^{e}=0$ for every $d \in R$ and $q^{\prime}=p^{e^{\prime}}$. Since $R \hookrightarrow S$ is a finite extension of normal domains, so $d S \cap R \neq 0$ for all nonzero elements $d \in S$. Therefore $c \cdot \bigcap_{e \geq e^{\prime}} \operatorname{Ker} d F_{S}^{e}=0$ for every $d \in S$ and $q^{\prime}=p^{e^{\prime}}$, namely, $c \in \tau\left(S, \Delta_{S}\right)$.

Hara and Yoshida informed the author of the restriction theorem and subadditivity theorem for the ideal " $\tau(R, \mathfrak{a})$ " (See [HY], Theorem 4.1 and Proposition 4.4]). Their results inspire him to obtain the restriction theorem and subadditivity theorem for " $\tau(R, \Delta)$ " which correspond to Proposition 1.9.

Proposition 2.12. (1) (Restriction Theorem) Let $(R, \mathfrak{m})$ be a complete normal Cohen-Macaulay $\mathbb{Q}$-Gorenstein local ring of characteristic $p>0$, and assume that the order of the canonical class in the divisor class group $\mathrm{Cl}(R)$ is not divisible by $p$. Let $\Delta$ be an effective $\mathbb{Q}$-Cartier divisor on $\operatorname{Spec} R$, that is, $r \Delta=\operatorname{div}_{R}(y)$ for some positive integer $r$ and nonzero element $y \in R$, and $x \in R$ be a nonzero divisor such that $R / x R$ is normal and $y \notin x R$. Then, denoting $S:=R / x R$, we have

$$
\tau\left(S,\left.\Delta\right|_{\text {Spec } S}\right) \subseteq \tau(R, \Delta) \cdot S .
$$

(2) (Subadditivity Theorem) Let $(R, \mathfrak{m})$ be a complete regular local ring of characteristic $p>0$, and $\Delta_{1}$ and $\Delta_{2}$ be any two effective $\mathbb{Q}$-divisors on $\operatorname{Spec} R$. Then

$$
\tau\left(R, \Delta_{1}+\Delta_{2}\right) \subseteq \tau\left(R, \Delta_{1}\right) \cdot \tau\left(R, \Delta_{2}\right)
$$

Proof. (1) We identify $E_{S}$ with $(0: x)_{E_{R}}$.

Claim 1.

$$
0_{E_{R}}^{* \Delta} \cap E_{S} \subseteq 0_{E_{S}}^{\left.* \Delta\right|_{\text {Spec } S}}
$$

Proof of Claim 1. First we will look at the Frobenius actions on $E_{R}$ and $E_{S}$. Since $R$ is Cohen-Macaulay, we have the following commutative diagram with exact rows 
for infinitely many $q=p^{e}$ (See the proof of $[\mathrm{HW}$, Theorem 4.9]).

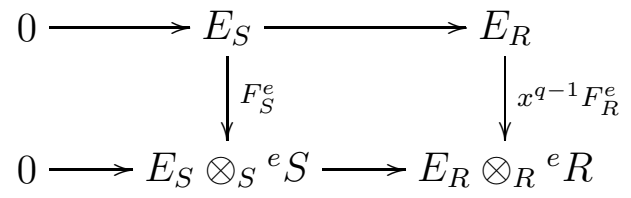

We fix sufficiently large such $q=p^{e}$. If $\xi \in 0_{E_{R}}^{* \Delta} \cap E_{S}$, then for some nonzero element $c \in R$, we have $c F_{R}^{e}(\xi)=0$ in $E_{R} \otimes_{R}{ }^{e} R((q-1) \Delta)$. We write $q-1=k r+i$ for integers $k$ and $i$ with $0 \leq i \leq r-1$. Then there exists a nonzero element $c^{\prime} \in R$ such that $c^{\prime} \notin x R$ and $c^{\prime} x^{q-1} y^{k} F_{R}^{e}(\xi)=0$ in $E_{R} \otimes_{R}{ }^{e} R$. Hence, by the above diagram, $c^{\prime} y^{k} F_{S}^{e}(\xi)=0$ in $E_{S} \otimes_{S}{ }^{e} S$, so that $c^{\prime} F_{S}^{e}(\xi)=0$ in $E_{S} \otimes_{S}{ }^{e} S\left(\left.(q-1) \Delta\right|_{\operatorname{Spec} S}\right)$ for infinitely many $q=p^{e}$. Since $c^{\prime} \notin x R$, it implies that $\xi \in 0_{E_{S}}^{\left.* \Delta\right|_{\operatorname{Spec} S}}$.

Since $R$ is complete, by [Ha3], Lemma 3.3], we have $0_{E_{R}}^{* \Delta}=(0: \tau(R, \Delta))_{E_{R}}$. Thus

$$
\begin{aligned}
(0: x)_{0_{E_{R}}^{* \Delta}} & =(0: \tau(R, \Delta)+x R)_{E_{R}}=\left(0: \frac{\tau(R, \Delta)+x R}{x R}\right)_{E_{S}} \\
& =(0: \tau(R, \Delta) \cdot S)_{E_{S}} .
\end{aligned}
$$

In light of Claim $1, \tau(R, \Delta) \cdot S=\operatorname{Ann}_{S}(0: x)_{0_{E_{R}}^{* \Delta}} \supseteq \tau\left(S,\left.\Delta\right|_{\operatorname{Spec} S}\right)$.

(2) First we consider the following claim.

Claim 2. Let $R=k\left[\left[x_{1}, \ldots, x_{n}\right]\right]$ (resp. $S=k\left[\left[y_{1}, \ldots, y_{m}\right]\right]$ ) be an $n$-dimensional (resp. $m$-dimensional) complete regular local ring over a field $k$ of characteristic $p>0$ and $\Delta_{R}$ (resp. $\left.\Delta_{S}\right)$ an effective $\mathbb{Q}$-Weil divisor on $\operatorname{Spec} R(\operatorname{resp}$. Spec $S$ ). Let $T=R \hat{\otimes}_{k} S=k\left[\left[x_{1}, \ldots, x_{n}, y_{1}, \ldots, y_{m}\right]\right]$, and we denote by $p_{R}: \operatorname{Spec} T \rightarrow \operatorname{Spec} R$ and $p_{S}: \operatorname{Spec} T \rightarrow \operatorname{Spec} S$ natural projections. Then

$$
\tau\left(T, p_{R}^{*} \Delta_{R}+p_{S}^{*} \Delta_{S}\right) \subseteq\left(\tau\left(R, \Delta_{R}\right) \otimes_{k} \tau\left(S, \Delta_{S}\right)\right) T .
$$

Proof of Claim 2. It suffices to show that

$$
0_{E_{T}}^{*\left(p_{R}^{*} \Delta_{R}+p_{S}^{*} \Delta_{S}\right)} \supseteq 0_{E_{R}}^{* \Delta_{R}} \otimes_{k} E_{S}+E_{R} \otimes_{k} 0_{E_{S}}^{* \Delta_{S}},
$$

but it is clear since $E_{T}=E_{R} \otimes_{k} E_{S}$.

Let $\rho: T=R \hat{\otimes}_{k} R \rightarrow R$ be a diagonal map, and we denote by $p_{1}$ (resp. $p_{2}$ ) $:$ Spec $T \rightarrow$ Spec $R$ the first (resp. second) projection. Then the natural surjection $T \rightarrow T / \operatorname{Ker} \rho=R$ is a complete intersection, so it follows from the repeated application of the restriction theorem that $\tau\left(R, \Delta_{1}+\Delta_{2}\right) \subseteq \tau\left(T, p_{1}^{*} \Delta_{1}+p_{2}^{*} \Delta_{2}\right) \cdot R$. On the other hand, by Claim 2, $\tau\left(T, p_{1}^{*} \Delta_{1}+p_{2}^{*} \Delta_{2}\right) \cdot R \subseteq \tau\left(R, \Delta_{1}\right) \cdot \tau\left(R, \Delta_{2}\right)$. Therefore the assertion follows.

Theorem 2.13. Let $(R, \mathfrak{m})$ be an F-finite normal local ring of characteristic $p>0$ and $\Delta$ an effective $\mathbb{Q}$-Weil divisor on $Y=\operatorname{Spec} R$ such that $K_{Y}+\Delta$ is $\mathbb{Q}$-Cartier. Let $f: X \rightarrow Y=\operatorname{Spec} R$ be a proper birational morphism with $X$ normal. Then

$$
\tau(R, \Delta) \subseteq H^{0}\left(X, \mathcal{O}_{X}\left(\left\lceil K_{X}-f^{*}\left(K_{Y}+\Delta\right)\right\rceil\right)\right) .
$$


Proof. The essential idea of the proof is seen in [HW, Theorem 3.3] and [Wa]. Our proof consists of six steps.

(Step 1) Take any nonzero element $c \in \tau(R, \Delta)$, and fix a nonzero element $d \in$ $R(-\lceil\Delta\rceil)$. Then, for every $q^{\prime}=p^{e^{\prime}}>0$, we have $c \cdot \underset{e \geq e^{\prime}}{\cap} \operatorname{Ker} c d F^{e}=0$, where $F^{e}: E_{R} \rightarrow E_{R} \otimes_{R}^{e} R((q-1) \Delta)$ is the $e$-times Frobenius map induced on $E_{R}$. Since $E_{R}$ is Artinian, there exists $q^{\prime \prime}=p^{e^{\prime \prime}}$ such that $\cap_{e \geq e^{\prime}} \operatorname{Ker} c d F^{e}=\underset{e^{\prime \prime} \geq e \geq e^{\prime}}{\cap} \operatorname{Ker} c d F^{e}$. Let

$$
\varphi_{e}: \operatorname{Hom}_{R}\left(R((q-1) \Delta)^{1 / q}, R\right) \rightarrow \operatorname{Hom}_{R}(R, R)=R
$$

be an $R$-linear map induced by the $R$-linear map $R \stackrel{(c d)^{1 / q}}{\longrightarrow} R((q-1) \Delta)^{1 / q}$, and set $\varphi=\underset{e^{\prime \prime} \geq e \geq e^{\prime}}{\bigoplus} \varphi_{e}$. Since $c d F^{e}$ is the Matlis dual of $\varphi_{e}$, the condition that $c$. $\cap_{e^{\prime \prime} \geq e \geq e^{\prime}} \operatorname{Ker} c d F^{e}=0$ implies that $c \in \operatorname{Im} \varphi$. Hence, for every $e^{\prime \prime} \geq e \geq e^{\prime}$, there exist $c_{e} \in R$ and an $R$-module homomorphism $\phi_{e}{ }^{\prime}: R((q-1) \Delta)^{1 / q} \rightarrow R$ sending $(c d)^{1 / q}$ to $c_{e}$ such that $\sum_{e^{\prime \prime} \geq e \geq e^{\prime}} c_{e}=c$.

(Step 2) We will prove that $\left\lfloor\Delta-\operatorname{div}_{R}(c)\right\rfloor \leq 0$. Assume to the contrary that $\Delta$ has a component $\Delta_{0}$ such that the coefficient of $\Delta$ in $\Delta_{0}$ is at least $1+v_{\Delta_{0}}(c)$, where $v_{\Delta_{0}}$ is the valuation of $\Delta_{0}$. Since the coefficient of $(q-1) \Delta+\operatorname{div}_{R}(d) \geq q \Delta$ in $\Delta_{0}$ is $q\left(1+v_{\Delta_{0}}(c)\right)$ or more, the $R$-linear map $R \stackrel{(c d)^{1 / q}}{\longrightarrow} R((q-1) \Delta)^{1 / q}$ factors through $R \hookrightarrow R\left(\left(1+v_{\Delta_{0}}(c)\right) \Delta_{0}\right)$. Hence, for every $e^{\prime \prime} \geq e \geq e^{\prime}, \phi_{e}{ }^{\prime}$ induces an $R$-module homomorphism $R\left(\left(1+v_{\Delta_{0}}(c)\right) \Delta_{0}\right) \rightarrow R$ which sends 1 to $c_{e}$. Thus there exists an $R$-linear map $R\left(\left(1+v_{\Delta_{0}}(c)\right) \Delta_{0}\right) \rightarrow R$ sending 1 to $c$. This is a contradiction.

(Step 3) Let $\phi_{e}=d^{1 / q} \phi_{e}{ }^{\prime}$. The $R$-module homomorphism $\phi_{e}$ (resp. $\left.\phi_{e}{ }^{\prime}\right)$ induces an $R$-linear map $\psi_{e}\left(\operatorname{resp} . \psi_{e}{ }^{\prime}\right): R\left((q-1) \Delta+\operatorname{div}_{R}(c)\right)^{1 / q} \rightarrow R\left(\operatorname{div}_{R}\left(c_{e}\right)\right)$ which sends 1 (resp. $\left.d^{1 / q}\right)$ to 1 . We may assume without loss of generality that $X$ is Gorenstein (cf. the proof of [HW, Theorem 3.3]). Thanks to the adjunction formula, we may regard $\psi_{e}\left(\right.$ resp. $\left.\psi_{e}^{\prime}\right)$ in

$$
\begin{aligned}
\operatorname{Hom}_{R}(R((q-1) \Delta & \left.\left.+\operatorname{div}_{R}(c)\right)^{1 / q}, R\left(\operatorname{div}_{R}\left(c_{e}\right)\right)\right) \\
& \cong R\left(\left\lceil(1-q)\left(K_{Y}+\Delta\right)+q \operatorname{div}_{R}\left(c_{e}\right)-\operatorname{div}_{R}(c)\right\rceil\right)^{1 / q}
\end{aligned}
$$

as a rational section of the sheaf $\mathcal{O}_{X}\left((1-q) K_{X}\right)$, and consider the corresponding divisor on $X$

$$
D_{e}=D_{\psi_{e}}=\left(\psi_{e}\right)_{0}-\left(\psi_{e}\right)_{\infty} \quad\left(\operatorname{resp} . D_{e}^{\prime}=D_{\psi_{e}}=\left(\psi_{e}^{\prime}\right)_{0}-\left(\psi_{e}^{\prime}\right)_{\infty}\right)
$$

where $\left(\psi_{e}\right)_{0}$ and $\left(\psi_{e}\right)_{\infty}$ (resp. $\left(\psi_{e}^{\prime}\right)_{0}$ and $\left.\left(\psi_{e}{ }^{\prime}\right)_{\infty}\right)$ are the divisors of zeros and poles of $\psi_{e}$ (resp. $\left.\psi_{e}^{\prime}\right)$ as rational sections of $\mathcal{O}_{X}\left((1-q) K_{X}\right)$. Clearly, $D_{e}=D_{e}{ }^{\prime}+\operatorname{div}_{X}(d)$. By definition, $D_{e}$ and $D_{e}{ }^{\prime}$ are linearly equivalent to $(1-q) K_{X}$, and $\left(\phi_{e}\right)_{\infty}$ and $\left(\phi_{e}{ }^{\prime}\right)_{\infty}$ are $f$-exceptional divisors. Hence $f_{*} D_{e}$ (resp. $f_{*} D_{e}{ }^{\prime}$ ) is linearly equivalent to $(1-q) K_{Y}$ and $f_{*} D_{e}\left(\operatorname{resp} . f_{*} D_{e}{ }^{\prime}\right) \geq\lfloor(q-1) \Delta\rfloor-q \operatorname{div}_{R}\left(c_{e}\right)+\operatorname{div}_{R}(c)$. We denote 
$X^{\prime}=X \backslash \operatorname{Supp}\left(\psi_{e}\right)_{\infty}$. Then $\psi_{e}$ lies in

$$
\begin{aligned}
& \operatorname{Hom}_{\mathcal{O}_{X^{\prime}}}\left(\mathcal{O}_{X^{\prime}}\left(\operatorname{div}_{X^{\prime}}(c)\right)^{1 / q}, \mathcal{O}_{X^{\prime}}\left(\operatorname{div}_{X^{\prime}}\left(c_{e}\right)\right)\right) \\
& \cong H^{0}\left(X^{\prime}, \mathcal{O}_{X^{\prime}}\left((1-q) K_{X^{\prime}}+q \operatorname{div}_{X^{\prime}}\left(c_{e}\right)-\operatorname{div}_{X^{\prime}}(c)\right)\right) .
\end{aligned}
$$

(Step 4) We will show that the coefficient of $D_{e}$ in each irreducible component is $q-1$ or less. Assume to the contrary that there exists an irreducible component $D_{e, 0}$ of $D_{e}$ whose coefficient is at least $q$. Let $v_{D_{e, 0}}$ be the valuation of $D_{e, 0}$ and $\alpha=q v_{D_{e, 0}}\left(c_{e}\right)-v_{D_{e, 0}}(c)+q$. Then $\psi_{e}$ lies in

$$
\begin{aligned}
H^{0}\left(X^{\prime}, \mathcal{O}_{X^{\prime}}\left(\left((1-q) K_{X^{\prime}}+q \operatorname{div}_{X^{\prime}}\left(c_{e}\right)-\operatorname{div}_{X^{\prime}}(c)\right)-\alpha D_{e, 0}\right)\right) \\
\cong \operatorname{Hom}_{\mathcal{O}_{X^{\prime}}}\left(\mathcal{O}_{X^{\prime}}\left(\alpha D_{e, 0}+\operatorname{div}_{X^{\prime}}(c)\right)^{1 / q}, \mathcal{O}_{X^{\prime}}\left(\operatorname{div}_{X^{\prime}}\left(c_{e}\right)\right)\right) .
\end{aligned}
$$

Therefore, we get the following commutative diagram.

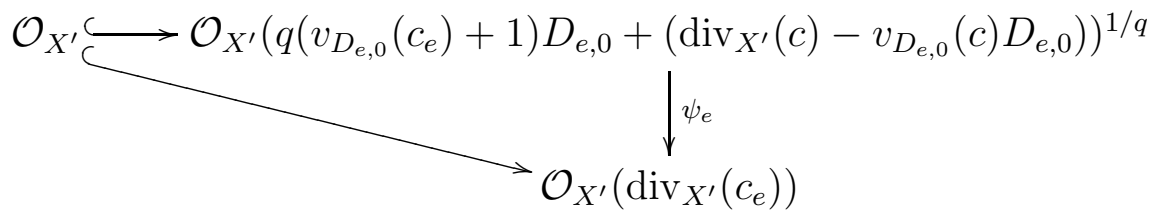

However the natural inclusion map

$$
\mathcal{O}_{X^{\prime}} \hookrightarrow \mathcal{O}_{X^{\prime}}\left(q\left(v_{D_{e, 0}}\left(c_{e}\right)+1\right) D_{e, 0}+\left(\operatorname{div}_{X^{\prime}}(c)-v_{D_{e, 0}}(c) D_{e, 0}\right)\right)^{1 / q}
$$

factors through $\mathcal{O}_{X^{\prime}}\left(\left(v_{D_{e, 0}}\left(c_{e}\right)+1\right) D_{e, 0}\right)$, and the above commutative diagram implies $\left(v_{D_{e, 0}}\left(c_{e}\right)+1\right) D_{e, 0} \leq \operatorname{div}_{X^{\prime}}\left(c_{e}\right)$. This is absurd. Hence every coefficient of $D_{e}$ in each irreducible component must be at most $q-1$.

(Step 5) We denote by $\cup_{j=1}^{s} E_{j}$ the exceptional divisor of $f$ and by $f_{*}^{-1} \Delta^{\prime}$ the strict transform of $\Delta^{\prime}:=\Delta-\operatorname{div}_{R}(c)$ in $X$. Then we write

$$
K_{X}+f_{*}^{-1} \Delta^{\prime} \underset{\mathbb{Q} \text {-lin. }}{\sim} f^{*}\left(K_{Y}+\Delta^{\prime}\right)+\sum_{j=1}^{s} a_{j} E_{j} .
$$

Let $B_{e}{ }^{\prime}=\frac{1}{q-1} D_{e}{ }^{\prime}-f_{*}^{-1} \Delta^{\prime}$. Then $B_{e}{ }^{\prime}$ is $\mathbb{Q}$-linearly equivalent to $-\left(K_{X}+f_{*}^{-1} \Delta^{\prime}\right)$, so that $f_{*} B_{e}{ }^{\prime}$ is $\mathbb{Q}$-linearly equivalent to $-f_{*}\left(K_{X}+f_{*}^{-1} \Delta^{\prime}\right)=-\left(K_{Y}+\Delta^{\prime}\right)$. Hence $f_{*} B_{e}{ }^{\prime}$ is $\mathbb{Q}$-Cartier. Since $B_{e}{ }^{\prime}+\sum_{j=1}^{s} a_{j} E_{j}$ is $\mathbb{Q}$-linearly equivalent to $-f^{*}\left(K_{Y}+\Delta^{\prime}\right)$, we know that $\left(B_{e}{ }^{\prime}-f^{*} f_{*} B_{e}{ }^{\prime}\right)+\sum_{j=1}^{s} a_{j} E_{j}$ is an $f$-exceptional divisor which is $\mathbb{Q}$-linearly trivial relative to $f$. Hence

$$
\left(B_{e}^{\prime}-f^{*} f_{*} B_{e}^{\prime}\right)+\sum_{j=1}^{s} a_{j} E_{j}=0 .
$$

(Step 6) Now, by (Step 3),

$$
\begin{aligned}
f_{*} D_{e}^{\prime}-(q-1) \Delta^{\prime} & \geq\left(\lfloor(q-1) \Delta\rfloor-q \operatorname{div}_{R}\left(c_{e}\right)+\operatorname{div}_{R}(c)\right)-(q-1) \Delta^{\prime} \\
& \geq-\Delta^{\prime \prime}+q\left(\operatorname{div}_{R}(c)-\operatorname{div}_{R}\left(c_{e}\right)\right)
\end{aligned}
$$


for some effective $\mathbb{Q}$-Cartier divisor $\Delta^{\prime \prime}$ on $Y$ which is independent of $q$. This implies $f_{*} B_{e}^{\prime} \geq-\frac{1}{q-1} \Delta^{\prime \prime}+\frac{q}{q-1}\left(\operatorname{div}_{R}(c)-\operatorname{div}_{R}\left(c_{e}\right)\right)$, whence

$$
f^{*} f_{*} B_{e} \geq-\frac{1}{q-1} f^{*} \Delta^{\prime \prime}+\frac{q}{q-1}\left(\operatorname{div}_{X}(c)-\operatorname{div}_{X}\left(c_{e}\right)\right) .
$$

On the other hand, we have seen in (Step 4) that the coefficient of $D_{e}$ in $E_{j}$ is at most $q-1$. Since $D_{e}=D_{e}{ }^{\prime}+\operatorname{div}_{X}(d)$ and we can assume that the coefficient of $\operatorname{div}_{X}(d)-f^{*} \Delta^{\prime \prime}$ in $E_{j}$ is greater than zero, the coefficient of $D_{e}^{\prime}+f^{*} \Delta^{\prime \prime}$ in $E_{j}$ is less than $q-1$. Hence the coefficient of $B_{e}{ }^{\prime}-f^{*} f_{*} B_{e}{ }^{\prime}$ in $E_{j}$ is less than $1-\frac{q}{q-1}\left(\operatorname{div}_{X}(c)-\right.$ $\left.\operatorname{div}_{X}\left(c_{e}\right)\right)$. Since $\sum_{e^{\prime \prime} \geq e \geq e^{\prime}} c_{e}=c$, for every $j=1, \ldots, s$, there exists $e^{\prime \prime} \geq e \geq e^{\prime}$ such that $v_{E_{j}}(c) \geq v_{E_{j}}\left(c_{e}\right)$, where $v_{E_{j}}$ is the valuation of $E_{j}$. Therefore via (Step 5) we have $a_{j}>-1$.

It follows from the above result and (Step 2) that

$$
\operatorname{div}_{X}(c)+\left\lceil K_{X}-f^{*}\left(K_{Y}+\Delta\right)=\left\lceil K_{X}-f^{*}\left(K_{Y}+\Delta^{\prime}\right)\right\rceil\right\rceil \geq 0,
$$

that is, $c \in H^{0}\left(X, \mathcal{O}_{X}\left(\left\lceil K_{X}-f^{*}\left(K_{Y}+\Delta\right)\right\rceil\right)\right)$.

Remark 2.14. By using the argument in (Step 1) of the proof of Theorem 2.13, we can also define the F-purity and F-regularity for ineffective divisors, and prove the similar results as those for effective divisors. However, the definition of F-purity and F-regularity for ineffective divisors is very complicated, therefore we only treat the effective case in this paper.

Example 2.15. (1) When $R$ is a regular local ring and $\Delta$ is an effective $\mathbb{Q}$-Weil divisor with simple normal crossing support, then $\tau(R, \Delta)=R(-\lfloor\Delta\rfloor)$.

(2) Let $R=k\left[\left[x_{1}, \ldots, x_{d}\right]\right]$ be a $d$-dimensional complete regular local ring over a field $k$ of characteristic $p>0$ and $\Delta=\operatorname{div}_{R}\left(x_{1}^{d+1}+\cdots+x_{d}^{d+1}\right)$. If the characteristic $p>d+1$, then $\tau\left(R, \frac{d}{d+1} \Delta\right)=\left(x_{1}, \ldots, x_{d}\right)$.

\section{Main Theorem}

To state the main result, we will explain the meaning of the phrase "in characteristic $p \gg 0$."

Let $R$ be a normal domain which is finitely generated over a field $k$ of characteristic zero and $\Delta$ an effective $\mathbb{Q}$-Weil divisor on Spec $R$ such that $K_{Y}+\Delta$ is $\mathbb{Q}$-Cartier. Let $f: X \rightarrow \operatorname{Spec} R$ be a resolution of singularities such that $\operatorname{Exc}(f)+f_{*}^{-1} \Delta$ has simple normal crossing support. Choosing a suitable finitely generated $\mathbb{Z}$-subalgebra $A$ of $k$, there exists a finitely generated normal flat $A$-algebra $R_{A}$, an effective $\mathbb{Q}$ Weil divisor $\Delta_{A}$ on Spec $R_{A}$, a smooth $A$-scheme $X_{A}$ and a birational $A$-morphism $f_{A}: X_{A} \rightarrow \operatorname{Spec} R_{A}$ such that $K_{R_{A}}+\Delta_{A}$ is $\mathbb{Q}$-Cartier, $\operatorname{Exc}\left(f_{A}\right)+f_{A_{*}}^{-1} \Delta_{A}$ has simple normal crossing support, and by tensoring $k$ over $A$ one gets back $R, \Delta, X$ and $f: X \rightarrow \operatorname{Spec} R$. Given a closed point $s \in \operatorname{Spec} A$ with residue field $\kappa=\kappa(s)$, we denote the corresponding fibers over $s$ by $f_{\kappa}: X_{\kappa} \rightarrow \operatorname{Spec} R_{\kappa}, \Delta_{\kappa}$, etc. Then the 
pairs $\left(R_{\kappa}, \Delta_{\kappa}\right)$ over general closed points $s \in \operatorname{Spec} A$ inherit the properties possessed by the original one $(R, \Delta)$.

Now we fix a general closed point $s \in \operatorname{Spec} A$ with residue field $\kappa=\kappa(s)$ of sufficiently large characteristic $p \gg 0$. Then we refer to the fibers over $s \in \operatorname{Spec} A$ as "reduction modulo $p \gg 0$," and use the phrase "in characteristic $p \gg 0$ " when we look at general closed fibers which are reduced from characteristic zero to characteristic $p \gg 0$ as above.

The following lemma is essential to prove "F-properties" in characteristic $p \gg 0$.

Lemma 3.1 ([Ha2, Proposition 3.6, Corollary 3.8]). Let $(R, \mathfrak{m})$ be a normal local ring of dimension $d \geq 2$, essentially of finite type over a perfect field $\kappa$ of characteristic $p>0$. Let $f: X \rightarrow \operatorname{Spec} R$ be a resolution of singularities and $D$ an $f$-ample $\mathbb{Q}$-Cartier divisor on $X$ with simple normal crossing support. We denote the closed fiber of $f$ by $Z$. If $(R, \mathfrak{m})$ is the localization at any prime ideal of a finitely generated $\kappa$-algebra which is a reduction modulo $p \gg 0$ as well as $X, D$ and $f: X \rightarrow \operatorname{Spec} R$, then the e-times Frobenius map

$$
F^{e}: H_{Z}^{d}\left(X, \mathcal{O}_{X}(-D)\right) \rightarrow H_{Z}^{d}\left(X, \mathcal{O}_{X}(-q D)\right)
$$

is injective for every $q=p^{e}$.

Now we state our main result.

Theorem 3.2. Let $(R, \mathfrak{m})$ be a normal local ring essentially of finite type over a field of characteristic zero and $\Delta$ an effective $\mathbb{Q}$-Weil divisor on $Y=\operatorname{Spec} R$ such that $K_{Y}+\Delta$ is $\mathbb{Q}$-Cartier. Then, in characteristic $p \gg 0$,

$$
\tau(R, \Delta)=\mathcal{J}(Y, \Delta)
$$

Proof. Thanks to Theorem 2.13, it suffices to prove that $\tau(R, \Delta) \supseteq \mathcal{J}(Y, \Delta)$ in characteristic $p \gg 0$. Let $f: X \rightarrow Y=$ Spec $R$ be a resolution of singularities such that $\operatorname{Exc}(f)+f_{*}^{-1} \Delta$ has simple normal crossing support.

Take a nonzero element $c \in R\left(-\Delta_{\text {red }}\right)$ such that $R_{c}$ is regular, where $\Delta_{\text {red }}$ is the reduced divisor whose support is equal to that of $\Delta$. Let $\Delta^{\prime}=\operatorname{div}_{R}(c)$. Then there is a rational number $0 \leq \epsilon \ll 1$ such that $\left\lfloor f^{*}\left(K_{Y}+\Delta\right)\right\rfloor=\left\lfloor f^{*}\left(K_{Y}+\Delta+\epsilon \Delta^{\prime}\right)\right\rfloor$. Take an $f$-ample $\mathbb{Q}$-Cartier divisor $H$ on $X$ which is supported on the exceptional locus of $f$ such that $\left\lfloor f^{*}\left(K_{Y}+\Delta+\epsilon \Delta^{\prime}\right)-H\right\rfloor=\left\lfloor f^{*}\left(K_{Y}+\Delta\right)\right\rfloor$. Set $D=H-f^{*}\left(K_{Y}+\Delta+\epsilon \Delta^{\prime}\right)$ and we may assume that $\operatorname{Exc}(f)+f_{*}^{-1}\left(\Delta+\epsilon \Delta^{\prime}\right)$ has simple normal crossing support again, replacing $f$ suitably. By Lemma 3.1, in characteristic $p \gg 0$, the $e$-times Frobenius map

$$
F^{e}: H_{Z}^{d}\left(X, \mathcal{O}_{X}\left(f^{*}\left(K_{Y}+\Delta\right)\right)\right) \rightarrow H_{Z}^{d}\left(X, \mathcal{O}_{X}(-q D)\right)
$$

is injective for every $q=p^{e}$, where $Z$ is the closed fiber of $f$.

On the other hand, let

$$
\delta: H_{\mathfrak{m}}^{d}\left(R\left(K_{Y}\right)\right) \rightarrow H_{Z}^{d}\left(X, \mathcal{O}_{X}\left(f^{*}\left(K_{Y}+\Delta\right)\right)\right)
$$


be the Matlis dual of the natural inclusion map

$$
\mathcal{J}(Y, \Delta)=H^{0}\left(X, \mathcal{O}_{X}\left(\left\lceil K_{X}-f^{*}\left(K_{Y}+\Delta\right)\right\rceil\right)\right) \hookrightarrow R
$$

and

$$
\delta_{e}: H_{\mathfrak{m}}^{d}\left(R\left(q\left(K_{Y}+\Delta+\epsilon \Delta^{\prime}\right)\right)\right) \rightarrow H_{Z}^{d}\left(X, \mathcal{O}_{X}\left(-q H+q f^{*}\left(K_{Y}+\Delta+\epsilon \Delta^{\prime}\right)\right)\right)
$$

the natural map induced by an edge map of the Leray spectral sequence

$$
H_{\mathfrak{m}}^{j}\left(H^{i}\left(X, \mathcal{O}_{X}\left(q f^{*}\left(K_{Y}+\Delta+\epsilon \Delta^{\prime}\right)\right)\right)\right) \Rightarrow H_{Z}^{i+j}\left(X, \mathcal{O}_{X}\left(q f^{*}\left(K_{Y}+\Delta+\epsilon \Delta^{\prime}\right)\right)\right) .
$$

Then by the Matlis duality,

$$
\begin{aligned}
\operatorname{ker}(\delta) & =\operatorname{Hom}_{R}\left(\frac{R}{\mathcal{J}(Y, \Delta)}, E_{R}\right)=\operatorname{Ann}_{H_{\mathfrak{m}}^{d}\left(R\left(K_{Y}\right)\right)} \mathcal{J}(Y, \Delta), \\
\operatorname{ker}\left(\delta_{e}\right) & =\operatorname{Hom}_{R}\left(\frac{R\left(\left\lceil K_{Y}-q\left(K_{Y}+\Delta+\epsilon \Delta^{\prime}\right)\right\rceil\right)}{H^{0}\left(X, \mathcal{O}_{X}\left(\left\lceil K_{X}+q D\right\rceil\right)\right)}, E_{R}\right) \\
& =\operatorname{Ann}_{H_{\mathfrak{m}}^{d}\left(R\left(q\left(K_{Y}+\Delta+\epsilon \Delta^{\prime}\right)\right)\right)} H^{0}\left(X, \mathcal{O}_{X}\left(\left\lceil K_{X}+q D\right\rceil\right)\right) .
\end{aligned}
$$

We obtain the following commutative diagram with exact rows for every $q=p^{e}$.

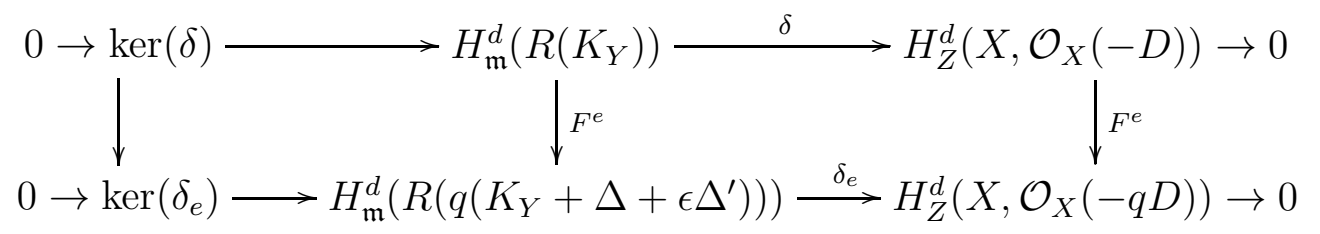

Take any element $\xi \in H_{\mathfrak{m}}^{d}\left(R\left(K_{Y}\right)\right) \backslash \operatorname{ker}(\delta)$. By the above diagram, $\xi^{q} \notin \operatorname{ker}\left(\delta_{e}\right)$. By Lemma 2.5, $c^{n}$ is a $\Delta$-test element for some positive integer $n$, and for sufficiently large $q$,

$$
\begin{aligned}
H^{0}\left(X, \mathcal{O}_{X}\left(\left\lceilK_{X}+q\right.\right.\right. & \left.\left.\left.\left(H-f^{*}\left(K_{Y}+\Delta+\epsilon \Delta^{\prime}\right)\right)\right\rceil\right)\right) \\
& \subseteq c^{n+1} H^{0}\left(X, \mathcal{O}_{X}\left(\left\lceil K_{X}+q\left(H-f^{*}\left(K_{Y}+\Delta\right)\right)\right\rceil\right)\right)
\end{aligned}
$$

Hence

$$
c^{n+1} \xi^{q} \notin \operatorname{Ann}_{H_{\mathfrak{m}}^{d}\left(R\left(q\left(K_{Y}+\Delta\right)\right)\right)} H^{0}\left(X, \mathcal{O}_{X}\left(\left\lceil K_{X}+q\left(H-f^{*}\left(K_{Y}+\Delta\right)\right)\right\rceil\right)\right) .
$$

If $\xi \in 0_{H_{\mathfrak{m}}^{d}\left(R\left(K_{Y}\right)\right)}^{* \Delta}$, then by the proof of Lemma 2.5, we have $c^{n} \xi^{q}=0$ in $H_{\mathfrak{m}}^{d}\left(R\left(\left\lceil q K_{Y}+\right.\right.\right.$ $q \Delta\rceil))$. Therefore $c^{n+1} \xi^{q}=0$ in $H_{\mathfrak{m}}^{d}\left(R\left(q\left(K_{Y}+\Delta\right)\right)\right)$, and this is a contradiction. It follows that

$$
0_{H_{\mathfrak{m}}^{d}\left(R\left(K_{Y}\right)\right)}^{* \Delta} \subseteq \operatorname{ker}(\delta)=\operatorname{Ann}_{H_{\mathfrak{m}}^{d}\left(R\left(K_{Y}\right)\right)} \mathcal{J}(Y, \Delta),
$$

and by Matlis duality (see [Ha3, Lemma 3.3]), $\tau(R, \Delta) \supseteq \mathcal{J}(Y, \Delta)$.

Remark 3.3. When $\Delta=0$, Theorem 3.2 coincides with the results of Hara [Ha3] and Smith [Sm2]. However, since $R$ is not necessarily $\mathbb{Q}$-Gorenstein in our situation, we cannot use their strategy which is to reduce the case where $R$ is quasi-Gorenstein by passing to an index one cover.

As a direct consequence of the main theorem, we get the equivalence of klt pairs and strongly F-regular pairs. 
Corollary 3.4 ([世W, Conjecture 5.1.1]). Let $(R, \mathfrak{m})$ be a normal local ring essentially of finite type over a field of characteristic zero and $\Delta$ an effective $\mathbb{Q}$-Weil divisor on $Y=\operatorname{Spec} R$ such that $K_{Y}+\Delta$ is $\mathbb{Q}$-Cartier. Then, $(Y, \Delta)$ is klt if and only if $(R, \Delta)$ is of strongly F-regular type.

Hara and K.-i. Watanabe [HW, Problem 5.1.2] conjectured that $(Y, \Delta)$ is lc if and only if $(R, \Delta)$ is of dense F-pure type. The following result about log canonical thresholds is a piece of evidence for their conjecture. See [Ko] for the basic properties of $\log$ canonical thresholds.

Corollary 3.5 ([HW, Conjecture 5.2.1]). Let $Y$ be a normal variety in characteristic zero with only klt singularity at a point $y \in Y$ and $\Delta$ be an effective $\mathbb{Q}$-Cartier divisor on $Y$. We denote by $C_{y}(Y, \Delta)$ the $\log$ canonical threshold of $\Delta$ at $y \in Y$, that is,

$$
\begin{aligned}
C_{y}(Y, \Delta) & =\sup \left\{t \in \mathbb{R}_{>0} \mid(Y, t \Delta) \text { is lc at } y \in Y\right\} \\
& =\sup \left\{t \in \mathbb{R}_{>0} \mid(Y, t \Delta) \text { is klt at } y \in Y\right\} .
\end{aligned}
$$

Then,

$$
\begin{aligned}
C_{y}(Y, \Delta) & =\sup \left\{t \in \mathbb{Q}_{>0} \mid\left(\mathcal{O}_{Y, y}, t \Delta\right) \text { is of dense F-pure type }\right\} \\
& =\sup \left\{t \in \mathbb{Q}_{>0} \mid\left(\mathcal{O}_{Y, y}, t \Delta\right) \text { is of strongly F-regular type }\right\} .
\end{aligned}
$$

Proof. By [HW, Theorem 3.7], the pair of dense F-pure type is lc. Hence the assertion is clear.

\section{REFERENCES}

[AM] I. Aberbach and B. MacCrimmon, Some results on test ideals, Proc. Edinburgh Math. Soc. (2) 42 (1999), 541-549.

[BS] J. Briançon and H. Skoda, Sur la clôture intégrale d'un idéal de germes de fonctions holomorphes en un point de $C^{n}$, C. R. Acad. Sci. Paris. Sér. A 278 (1974), 949-951.

[DEL] J.-P. Demailly, L. Ein and R. Lazarsfeld, A subadditivity property of multiplier ideals, Michigan. Math. J. 48 (2000), 137-156.

[ELS] L. Ein, R. Lazarsfeld, and K. Smith, Uniform bounds and symbolic powers on smooth varieties, Invent. Math. 144 (2001), 241-252.

[Fe] R. Fedder, F-purity and rational singularity, Trans. Amer. Math. Soc. 278 (1983), 461-480.

[Ha1] N. Hara, F-regularity and F-purity of graded rings, J. Algebra, 172 (1995), 804-818.

[Ha2] — A characterization of rational singularities in terms of injectivity of Frobenius maps, Amer. J. Math. 120 (1998), 981-996.

[Ha3] _ Geometric interpretation of tight closure and test ideals, Trans. Amer. Math. Soc. 353 (2001), 1885-1906.

[HW] N. Hara and K.-i. Watanabe, F-regular and F-pure rings vs. log terminal and log canonical singularities, J. Alg. Geom. 11 (2002), 363-392.

[HY] N. Hara and K. Yoshida, A generalization of tight closure and multiplier ideals, Trans. Amer. Math. Soc. (to appear).

[HH1] M. Hochster and C. Huneke, Tight closure, invariant theory and the Briançon-Skoda theorem, J. Amer. Math. Soc. 3 (1990), 31-116.

[HH2] — Tight closure and strong F-regularity, Mem. Soc. Math. France 38 (1989), 119-133.

[HH3] C Comparison of symbolic and ordinary powers of ideals, Invent. Math. 147 (2002), 349-369. 
[HR] M. Hochster and J. Roberts, The purity of the Frobenius and local cohomology, Adv. Math. 21 (1976), 117-172.

[Hu] C. Huneke, "Tight closure and its applications," CBMS Regional Conf. Ser. Math. 88, Amer. Math. Soc., Providence (1996).

[Ko] J. Kollár, Singularities of pairs: in "Algebraic Geometry-Santa Cruz 1995", Proc. Symp. Pure Math. 62 (1997), 221-287.

[KM] J. Kollár and S. Mori, "Birational Geometry of Algebraic Varieties," Cambridge Tracts in Math. 134, Cambridge University Press, 1998.

[Ku] E. Kunz, On Noetherian rings of characteristic p, Amer. J. Math. 98 (1976), 999-1013.

[La] R. Lazarsfeld, Multiplier ideals for algebraic geometers, preprint.

[Mc] B. MacCrimmon, Weak F-regularity is strong F-regularity for rings with isolated non- $\mathbb{Q}$ Gorenstein points, Trans. Amer. Math. Soc. (to appear).

[MS] V. B. Mehta and V. Srinivas, A characterization of rational singularities, Asian. J. Math. 1 (1997), 249-278.

[Sm1] K. Smith, F-rational rings have rational singularities, Amer. J. Math. 119 (1997), 159-180.

[Sm2] - The multiplier ideal is a universal test ideal, Comm. Algebra 28 (2000), 5915-5929.

[Wa] K.-i. Watanabe, A characterization of "bad" singularities via the Frobenius map, Proceedings of the 18-th symposium on commutative algebra (Toyama, 1996), 122-126, 1996. (in Japanese).

[Wi] L. J. Williams, Uniform stability of kernels of Koszul cohomology indexed by the Frobenius endomorphism, J. Algebra 172 (1995), 721-743.

Graduate School of Mathematical Sciences, University of Tokyo, 3-8-1, Komaba, MEguro, TOKYO 153-8914, JAPAN

E-mail address: stakagi@ms.u-tokyo.ac.jp 